\title{
TWO DEFINABILITY RESULTS IN THE EQUATIONAL CONTEXT
}

\author{
M. HÉBERT, R. N. McKENZIE, AND G. E. WEAVER
}

(Communicated by Donald S. Passman)

\begin{abstract}
Let $\tau$ be a type bounded by an infinite regular cardinal $\alpha, \mathbf{V}$ be a variety in $\tau, \tau^{\prime} \subseteq \tau$ and $\mathbf{V}^{\prime}$ the class of all $\tau^{\prime}$-reducts of the algebras in $\mathbf{V}$. We show that the operations in $\tau \backslash \tau^{\prime}$ are explicitely definable in $\mathbf{V}$ by pure formulas (i.e. existential-positive without disjunction) if and only if they are implicitely definable and $\mathbf{V}^{\prime}$ is closed under unions of $\alpha$-chains (if and only if every $\tau^{\prime}$-homomorphisms between algebras in $\mathbf{V}$ are $\tau$-homomorphisms, as $\mathbf{J}$. Isbell has shown). It follows that the operations in $\tau \backslash \tau^{\prime}$ are equivalent (in $\mathbf{V}$ ) to $\tau^{\prime}$-terms if and only if every algebra in the $\left(\tau^{\prime}-\right)$ variety generated by $\mathbf{V}^{\prime}$ has a unique $\tau$-expansion in $\mathbf{V}$.
\end{abstract}

As usual, ordinals will be identified with the set of smaller ordinals, and cardinals with initial ordinals.

Definitions 1. (i) A type $\tau$ is a set of operation symbols, each with an assigned arity (some cardinal). $\tau$ is bounded by the infinite regular cardinal $\alpha$ if the arities of its operation symbols are all $<\alpha$.

(ii) If $\mathbf{C}$ is a class of ( $\tau-$ ) algebras, an $\alpha$-sequence (in $\mathbf{C}$ ) is an $\alpha$-chain $\left\{\mathfrak{A}_{\mu} \subseteq \mathfrak{A}_{\gamma} \mid 0 \leq \mu<\gamma<\alpha\right\}$ (where " $\subseteq$ " means "subalgebra") of algebras in $\mathbf{C}$ each of which is a section of a fixed $\mathfrak{A} \in \mathbf{C}$ (i.e. $\mathfrak{A}_{\mu} \subseteq \mathfrak{A}$ for every $\mu$ and these inclusions have left inverse homomorphisms).

(iii) If $\tau^{\prime} \subseteq \tau$ and $\mathfrak{A}$ is a $\tau$-algebra, we will denote, if the context is clear, by $\mathfrak{A}^{\prime}$ (instead of the usual $\mathfrak{A} \Gamma_{\tau^{\prime}}$ ) the $\tau^{\prime}$-reduct [2] of $\mathfrak{A}$. Similarly, if $\mathbf{C}$ is a class of $\tau$-algebras, $\mathbf{C}^{\prime}$ will be an abbreviation for $\mathbf{C} \Gamma_{\tau^{\prime}}=\left\{\mathfrak{A}^{\prime} \mid \mathfrak{A} \in \mathbf{C}\right\}$.

Lemma. Let $\tau$ be a type bounded by $\alpha, \mathbf{V}$ a variety of type $\tau$, and $\tau^{\prime} \subseteq \tau$. The following are equivalent:

(a) Every $\tau^{\prime}$-homomorphism between algebras in $\mathbf{V}$ is a $\tau$-homomorphism.

(b) For every $\alpha$-chain $\left\{\mathfrak{A}_{\mu}^{\prime} \subseteq \mathfrak{A}_{\gamma}^{\prime} \mid 0 \leq \mu<\gamma<\alpha\right\}$ in $\mathbf{V}^{\prime}, \bigcup_{\mu<\alpha} \mathfrak{A}_{\mu}^{\prime}$ is the $\tau^{\prime}$-reduct of a unique algebra in $\mathbf{V}$.

(c) Same as (b), with " $\alpha$-chain" replaced by " $\alpha$-sequence."

Received by the editors December 20, 1988.

1980 Mathematics Subject Classification (1985 Revision). Primary 08B05; Secondary 03C40, $08 \mathrm{C} 10$.

The first author received the financial support of the NSERC (Canada) and the FCAR (Québec). 
Proof. (a) $\Rightarrow$ (b) From (a), $\mathfrak{A} \subseteq \mathfrak{B}$ (in $\mathbf{V}$ ) if and only if $\mathfrak{A}^{\prime} \subseteq \mathfrak{B}^{\prime}$. Note also that (a) implies that distinct algebras in $\mathbf{V}$ have distinct $\tau^{\prime}$-reducts. This gives the result, as $\mathbf{V}$ is closed under unions of all $\alpha$-chains.

(b) $\Rightarrow$ (c) . Trivial.

(c) $\Rightarrow$ (a). Let $f: \mathfrak{A}^{\prime} \rightarrow \mathfrak{B}^{\prime}$ be a $\left(\tau^{\prime}-\right)$ homomorphism, $\mathfrak{A}, \mathfrak{B} \in \mathbf{V}$. We consider the $\alpha$ th power $\mathfrak{C}=(\mathfrak{A} \times \mathfrak{B})^{\alpha}$ of $(\mathfrak{A} \times \mathfrak{B})$, and it subset $D=$ $\{q: \alpha \rightarrow A \times B \mid$ there exist $\mu<\alpha$ and $a \in A$ such that $q(\gamma)=\langle a, f(a)\rangle$ for every $\gamma \geq \mu\}$. It follows from the fact that $\tau^{\prime}$ is bounded by $\alpha$ that $D$ is closed (in $\mathfrak{C}$ ) under the operations in $\tau^{\prime}$; hence it is the underlying set of a $\operatorname{sub}\left(\tau^{\prime}-\right)$ algebra $\mathfrak{D}^{0}$ of $\mathfrak{C}^{\prime}$.

The only thing we need to show here is that $D$ is closed in $\mathfrak{C}$ under all the operations in $\tau$. Indeed, for a $\gamma$-ary operation symbol $F$ in $\tau \backslash \tau^{\prime}$, we must prove that for every $\gamma$-tuple $\left\langle a_{\mu}\right\rangle_{\mu<\gamma}$ of elements of $A, f\left(F^{\mathfrak{A}}\left(\left\langle a_{\mu}\right\rangle_{\mu<\gamma}\right)\right)=$ $F^{\mathfrak{B}}\left(\left\langle f\left(a_{\mu}\right)\right\rangle_{\mu<\gamma}\right)$; consider the $\gamma$-tuple $\left\langle q_{\mu}\right\rangle_{\mu<\gamma}$ of elements of $D$ where for each $\mu<\gamma, q_{\mu}: \alpha \rightarrow A \times B$ is the constant function defined by $q_{\mu}(\beta)=\left\langle a_{\mu}, f\left(a_{\mu}\right)\right\rangle$ for every $\beta<\alpha$. If $D$ is closed under $F$ in $\mathfrak{C}$, then $F^{\mathfrak{C}}\left(\left\langle q_{\mu}\right\rangle_{\mu<\gamma}\right) \in D$, which means that there exist $\beta<\alpha$ and $a \in A$ such that $F^{\mathfrak{C}}\left(\left\langle q_{\mu}\right\rangle_{\mu<\gamma}\right)(\delta)=$ $\langle a, f(a)\rangle$ for every $\delta \geq \beta$. But the definition of products in $\mathbf{V}$ implies that $F^{\mathfrak{C}}\left(\left\langle q_{\mu}\right\rangle_{\mu<\gamma}\right)(\delta)=\left\langle F^{\mathfrak{A}}\left(\left\langle a_{\mu}\right\rangle_{\mu<\gamma}\right), F^{\mathfrak{B}}\left(\left\langle f\left(a_{\mu}\right)\right\rangle_{\mu<\gamma}\right)\right\rangle$ for every $\delta<\alpha$. This shows that $f\left(F^{\mathfrak{A}}\left(\left\langle a_{\mu}\right\rangle_{\mu<\gamma}\right)\right)=F^{\mathfrak{B}}\left(\left\langle f\left(a_{\mu}\right)\right\rangle_{\mu<\gamma}\right)$ as required.

Notice that $\prod_{x \in X}\left(\mathfrak{C}_{x}^{\prime}\right)=\left(\prod_{x \in X} \mathfrak{C}_{x}\right)^{\prime}$ for any set $X$ and any $\mathfrak{C} \in \mathbf{V}$. Consider the chain of injective $\tau^{\prime}$-homomorphisms $\left\{\lambda_{\mu, \gamma}:\left(\mathfrak{A}^{\prime} \times \mathfrak{B}^{\prime}\right)^{\mu} \times \mathfrak{A}^{\prime} \rightarrow\left(\mathfrak{A}^{\prime} \times \mathfrak{B}^{\prime}\right)^{\gamma} \times\right.$ $\left.\mathfrak{A}^{\prime} \mid 0 \leq \mu<\gamma<\alpha\right\}$, where $\lambda_{\mu, \gamma}=1_{\mu} \times f^{*}, 1_{\mu}$ is the identity homomorphism on $\left(\mathfrak{A}^{\prime} \times \mathfrak{B}^{\prime}\right)^{\mu}$ and $f^{*}: \mathfrak{A}^{\prime} \rightarrow\left(\mathfrak{A}^{\prime} \times \mathfrak{B}^{\prime}\right)^{\gamma \backslash \mu} \times \mathfrak{A}^{\prime}$ is the homomorphism induced (as a homomorphism into a product) by as many repetitions as required of $1_{\mathfrak{A}}$ (the identity on $\mathfrak{A}$ ) and $f$. It is easily seen that for every $\mu<\alpha$, the homomorphism $\lambda_{\mu}:\left(\mathfrak{A}^{\prime} \times \mathfrak{B}^{\prime}\right)^{\mu} \times \mathfrak{A}^{\prime} \rightarrow\left(\mathfrak{A}^{\prime} \times \mathfrak{B}^{\prime}\right)^{\alpha}$ determined by as many as required $1_{\mathfrak{A}}$ 's and $f$ 's has a left inverse which is a homomorphism (for example the projection onto the initial string of direct factors) and satisfies $\lambda_{\gamma} \cdot \lambda_{\mu, \gamma}=\lambda_{\mu}$ for every $\mu<\lambda<\alpha$. This means that $\left\{\lambda_{\mu, \gamma} \mid 0 \leq \mu<\gamma<\alpha\right\}$ is, "up to isomorphisms," an $\alpha$-sequence in $\mathbf{V}^{\prime}$. Clearly, $\mathfrak{D}^{0}=\bigcup_{\mu<\alpha}\left(\left(\mathfrak{A}^{\prime} \times \mathfrak{B}^{\prime}\right)^{\mu} \times \mathfrak{A}^{\prime}\right)($ via the identification under the above isomorphisms). By the hypothesis, $\mathfrak{D}^{0}=\mathfrak{D}^{\prime}$ for a unique $\mathfrak{D} \in \mathbf{V}$. It remains to show that $\mathfrak{D} \subseteq \mathfrak{C}$.

From now on, we will assume, for clarity, that $\alpha=\omega$ and $F$ is 1-ary (our argument will clearly extend to the general case). Note first that the hypotheses imply that any algebra $\mathfrak{C}^{\prime} \in \mathbf{V}^{\prime}$ is the $\tau^{\prime}$-reduct of only one algebra in $\mathbf{V}$ (consider the trivial $\alpha$-sequence $\left.\mathfrak{C}^{\prime} \subseteq \mathfrak{C}^{\prime} \subseteq \cdots\right)$. Next, we remark that for every $n<\omega$, the bijection $s_{n}: D \rightarrow(A \times B)^{n} \times D$ defined by

$$
s_{n}\left(\left\langle a_{0}, b_{1}, a_{2}, \ldots\right\rangle\right)=\left\langle\left\langle a_{0}, b_{1}, a_{2}, \ldots, b_{2 n-1}\right\rangle,\left\langle a_{2 n}, b_{2 n+1}, \ldots\right\rangle\right\rangle
$$


is a $\tau^{\prime}$-homomorphism from $\mathfrak{D}^{\prime}$ to $\left(\mathfrak{A}^{\prime} \times \mathfrak{B}^{\prime}\right)^{n} \times \mathfrak{D}^{\prime}\left(=\left((\mathfrak{A} \times \mathfrak{B})^{n} \times \mathfrak{D}\right)^{\prime}\right)$. Now, any $\tau^{\prime}$-isomorphism $g$ from the $\tau^{\prime}$-reduct $\mathfrak{D}^{\prime}$ of a $\tau$-algebra $\mathfrak{D}$ to a $\tau^{\prime}$-algebra $\mathfrak{C}^{0}$ determines a unique $\tau$-structure $\mathfrak{C}$ such that $\mathfrak{C}^{\prime}=\mathfrak{C}^{0}$ and $g$ is a $\tau$-isomorphism from $\mathfrak{D}$ to $\mathfrak{C}$. But $\left((\mathfrak{A} \times \mathfrak{B})^{n} \times \mathfrak{D}\right)^{\prime}$ is the $\tau^{\prime}$-reduct of a unique algebra in $\mathbf{V}$, and then $s_{n}$ is a $(\tau-)$ isomorphism from $\mathfrak{D}$ to $\left((\mathfrak{A} \times \mathfrak{B})^{n} \times \mathfrak{D}\right)$. In particular, we have that $s_{n}\left(F^{\mathcal{D}}\left(\left\langle a_{0}, b_{1}, a_{2}, \ldots\right\rangle\right)=F^{\left((\mathfrak{A} \times \mathfrak{B})^{n} \times \mathfrak{D}\right.}\left(\left\langle a_{0}, b_{1}, a_{2}, \ldots\right\rangle\right)=\right.$ $\left\langle\left\langle F^{\mathfrak{A}}\left(a_{0}\right), F^{\mathfrak{B}}\left(b_{1}\right), F^{\mathfrak{A}}\left(a_{2}\right), \ldots, F^{\mathfrak{B}}\left(b_{2 n-1}\right)\right\rangle, F^{\mathfrak{D}}\left(\left\langle a_{2 n}, b_{2 n+1}, \ldots\right\rangle\right)\right\rangle$. This being true for all $n$, we have, by the very definition of $s_{n}$, that $F^{\mathfrak{D}}\left(\left\langle a_{0}, b_{1}, a_{2}, \ldots\right\rangle\right)=$ $\left\langle F^{\mathfrak{A}}\left(a_{0}\right), F^{\mathfrak{B}}\left(b_{1}\right), F^{\mathfrak{A}}\left(a_{2}\right), \ldots\right\rangle$, as required.

Remark. The following facts, which can be extracted from the proof of (c) $\Rightarrow$ (a) above, will be used later: (1) if $f: \mathfrak{A}^{\prime} \rightarrow \mathfrak{B}^{\prime}$ is a $\left(\tau^{\prime}-\right.$ ) homomorphism, $\mathfrak{A}, \mathfrak{B} \in \mathbf{V}$, then $f: \mathfrak{A} \rightarrow \mathfrak{B}$ is a $(\tau-)$ homomorphism if and only if $\mathfrak{D}^{0}$ (constructed as in the proof) is the $\tau$-reduct of a unique algebra in $\mathbf{V} ;(2) \mathfrak{D}^{0}$ is a subdirect product of repetitions of $\mathfrak{A}^{\prime}$ and $\mathfrak{B}^{\prime}$.

To place this result in a more general perspective and to point out some consequences, we need to recall the definitions of a few more concepts.

Definitions 2. (i) We denote by $L_{\alpha}(\tau)$ the language having the elements of $\tau$ as its operation symbols (no relation symbol other than $=$ ) and which contains all formulas constructed as usual from atomic formulas by quantification, disjunction and conjunction over sets of cardinality smaller than $\alpha$, and by negation and implication. Similar languages are sometimes denoted by $L_{\alpha, \alpha}(\tau)$ (see [2]). Here, unlike other accounts, infinitary operation symbols may occur in $\tau$. A formula is pure if it is of the form $\exists \mathbf{x}(\bigwedge \Phi(\mathbf{x}))$, where $\mathbf{x}$ is a string of variables and $\Phi(\mathbf{x})$ is a set of atomic formulas.

(ii) Let $\mathbf{C}$ be a class of $\tau$-algebras. We will write $\mathbf{M}\left(\mathbf{T H}_{\alpha}(\mathbf{C})\right)$ for the elementary closure of $\mathbf{C}$, i.e. the class of models of the set of $L_{\alpha}^{\alpha}(\tau)$-sentences true in all algebras in $\mathbf{C} . \mathbf{M}(\tau)$ denotes the class of all $\tau$-algebras. $\operatorname{Var}(\mathbf{C})$ will be the smallest $(\tau-)$ variety containing $\mathbf{C}$, and $\underline{\mathbf{C}}$ will be the category of all algebras in $\mathbf{C}$ with all the homomorphisms between them. We say that $\mathbf{C}$ is closed under equalizers (in $\mathbf{M}(\boldsymbol{\tau})$ ) if for all $f, g: \mathfrak{A} \rightarrow \mathfrak{B}$ in $\underline{\mathbf{C}}$, the subalgebra of $\mathfrak{A}$ on the set $\{a \in A \mid f(a)=g(a)\}$ is in C. It is closed under limits if it is closed under equalizers and products (in $(\mathbf{M}(\tau))$.

Theorem. Let $\tau=\tau^{\prime} \cup\{F\}$ be a type which is bounded by $\alpha$, and let $\mathbf{V}$ be a variety of $\tau$-algebras. If $\mathbf{C}$ is any class of $\tau^{\prime}$-algebras containing $\mathbf{V}^{\prime}$, we denote by $K: \mathbf{V} \rightarrow \mathbf{C}$ the mapping $\mathfrak{A} \mapsto \mathfrak{A}^{\prime}$, and by $\underline{K}: \underline{\mathbf{V}} \rightarrow \underline{\mathbf{C}}$ the corresponding functor.

I. If $\alpha=\omega$, the following are equivalent:

(a) $\mathbf{V} \vDash \forall \mathbf{x} y(F(\mathbf{x})=y \leftrightarrow \phi(\mathbf{x}, y))$ for some formula $\phi(\mathbf{x}, y)$ of $L_{c}\left(\tau^{\prime}\right)$.

(b) $K: \mathbf{V} \rightarrow \mathbf{V}^{\prime}$ is injective ( $\Leftrightarrow$ bijective).

(c) $K: \mathbf{V} \rightarrow \mathbf{M}\left(\mathbf{T H} \mathbf{\alpha}_{\alpha}\left(\mathbf{V}^{\prime}\right)\right)$ is bijective. 
II. The following are equivalent:

$(\mathbf{a})^{\prime} \mathbf{V} \vDash \forall \mathbf{x} y(F(\mathbf{x})=y \leftrightarrow \phi(\mathbf{x}, y))$ for some pure formula $\phi(\mathbf{x}, y)$ of $L_{\alpha}\left(\tau^{\prime}\right)$.

(b) ' $\underline{K}: \underline{\mathbf{V}} \rightarrow \underline{\mathbf{V}}^{\prime}$ is an isomorphism.

(c) ${ }^{\prime} \underline{K}: \underline{\mathbf{V}} \rightarrow \underline{\mathbf{M}}\left(\mathbf{T H}_{\alpha}\left(\underline{\mathbf{V}}^{\prime}\right)\right)$ is an isomorphism.

(d) ${ }^{\prime} \underline{K}: \underline{\mathbf{V}} \rightarrow \underline{\mathbf{V}^{\prime}}$ is full.

(e) ${ }^{\prime} K: \overline{\mathbf{V}} \rightarrow \overline{\mathbf{V}}^{\prime}$ is injective and $\mathbf{V}^{\prime}$ is closed (in $\mathbf{M}\left(\boldsymbol{\tau}^{\prime}\right)$ ) under unions of $\alpha$-sequences.

The following are equivalent and are consequences of the previous statements. They imply them if $\alpha=\omega$.

(f) ${ }^{\prime} K: \mathbf{V} \rightarrow \mathbf{V}^{\prime}$ is injective and $\mathbf{V}^{\prime}$ is closed (in $\mathbf{M}\left(\boldsymbol{\tau}^{\prime}\right)$ ) under equalizers.

$(\mathbf{g})^{\prime} \quad K: \mathbf{V} \rightarrow \mathbf{V}^{\prime}$ is injective and $\mathbf{V}^{\prime}$ is closed (in $\mathbf{M}\left(\boldsymbol{\tau}^{\prime}\right)$ ) under limits.

III. The following are equivalent:

(a) ${ }^{\prime \prime} \mathbf{V} \vDash \forall \mathbf{x}(F(\mathbf{x})=t(\mathbf{x}))$ for some $\tau^{\prime}$-term $t$.

(b) ${ }^{\prime \prime} \underline{K}: \underline{\mathbf{V}} \rightarrow \underline{\mathbf{V}}^{\prime}$ is an isomorphism and $\mathbf{V}^{\prime}$ has all freely generated algebras.

$\left(c_{1}\right)^{\prime \prime} K: \mathbf{V} \rightarrow \operatorname{Var}\left(\mathbf{V}^{\prime}\right)$ is bijective.

$\left(c_{2}\right)^{\prime \prime} \underline{K}: \underline{\mathbf{V}} \rightarrow \underline{\operatorname{Var}\left(\mathbf{V}^{\prime}\right)}$ is an isomorphism.

(d)" $\underline{K}: \underline{\mathbf{V}} \rightarrow \underline{\mathbf{V}^{\prime}}$ is full and $\mathbf{V}^{\prime}$ has the free algebra on $\gamma \quad$ (= arity of $F)$ generators.

(e) ${ }^{\prime \prime} K: \mathbf{V} \rightarrow \mathbf{V}^{\prime}$ is injective and $\mathbf{V}^{\prime}$ is a variety.

(f) ${ }^{\prime \prime} K: \mathbf{V} \rightarrow \mathbf{V}^{\prime}$ is injective and $\mathbf{V}^{\prime}$ is closed (in $\left.\mathbf{M}\left(\boldsymbol{\tau}^{\prime}\right)\right)$ under subalgebras.

(g) ${ }^{\prime \prime} \quad K: \mathbf{V} \rightarrow \mathbf{V}^{\prime}$ is injective and $\mathbf{V}^{\prime}$ is closed (in $\mathbf{M}\left(\boldsymbol{\tau}^{\prime}\right)$ ) under subdirect products.

(h) $" \underline{K}: \underline{\mathbf{V}} \rightarrow \underline{\operatorname{Var}\left(\mathbf{V}^{\prime}\right)}$ "creates monomorphisms" (that is, for every $\mathfrak{A} \in$ $\mathbf{V}$, every $\left(\tau^{\prime}-\right)$ subalgebra of $K(\mathfrak{A})$ extends to a $(\tau-)$ subalgebra of $\mathfrak{A})$.

Proof I. The equivalence of (a) and (b) is a special case of a result established by Beth (see [2, p. 87]). This equivalence is referred to here as the Beth Definability theorem. Likewise, the equivalence of (a) and (c) is a special case of a result of De Bouvère (see [3]). This equivalence is referred to here as the De Bouvère Definability theorem.

II. $(\text { a })^{\prime} \Leftrightarrow(\mathbf{d})^{\prime}$ This is shown by J. R. Isbell in [7].

$(\mathrm{d})^{\prime} \Leftrightarrow(\mathrm{b})^{\prime}$ Trivial, as $\underline{K}$ is always faithful.

$(\text { c })^{\prime} \Rightarrow(b)^{\prime}$ Obvious.

$(\mathbf{a})^{\prime} \Rightarrow(\mathbf{c})^{\prime} \quad$ From $(\mathbf{a})^{\prime}$, every $\tau$-identity true in $\mathbf{V}$ is equivalent (in $\mathbf{V}$ ) to a sentence of $L_{\alpha}\left(\tau^{\prime}\right)$; this implies that for any $\tau^{\prime}$-algebra $\mathfrak{A} \in \mathbf{M}\left(\mathbf{T} \mathbf{H}_{\alpha}\left(\mathbf{V}^{\prime}\right)\right)$, the $\tau$-algebra obtained from it by choosing for $F^{\mathfrak{a}}(\mathbf{a}), \mathbf{a} \in A^{\operatorname{arity}(F)}$, the unique $a \in A$ such that $\mathfrak{A} \vDash \phi[\mathbf{a}, a]$, is a model of the identities of $\mathbf{V}$, and hence is in 
$\mathbf{V}$. But clearly $(\mathbf{b})^{\prime}\left(\Leftrightarrow(\mathbf{a})^{\prime}\right)$ implies that the functor $\left.\underline{K}: \underline{\mathbf{V}} \rightarrow \underline{\mathbf{M}\left(\mathbf{T} \mathbf{H}_{\underline{\alpha}}\right.} \underline{\left(\mathbf{V}^{\prime}\right)}\right)$ is full, and hence an isomorphism.

$(\mathrm{d})^{\prime} \Leftrightarrow(\mathrm{e})^{\prime} \quad$ This is our lemma.

$(\mathrm{c})^{\prime} \Rightarrow(\mathrm{f})^{\prime} \quad$ This follows from the fact that $\mathbf{V}$ is itself closed (in $\mathbf{M}(\boldsymbol{\tau})$ ) under limits.

$(\mathrm{f})^{\prime} \Rightarrow(\mathrm{g})^{\prime} \quad$ Clear, as $\mathbf{V}^{\prime}$ is always closed under products.

$(\mathrm{g})^{\prime} \Rightarrow(\mathrm{e})^{\prime}$ This follows from I and the first two equivalences in Proposition 16 of [9].

III. $(\mathrm{g})^{\prime \prime} \Rightarrow(\mathrm{d})^{\prime \prime}$ Note first that by the remark following the lemma, $(\mathrm{g})^{\prime \prime}$ implies the equivalent statements of part III. Now, by Theorem 11.11 of [1], there exists a cardinal $\mu$ such that $\mathbf{V}^{\prime}$ has a free algebra on $\beta$ generators for all $\beta \geq \mu$. Let $\beta$ be a cardinal greater than $\mu$ and $\gamma(=$ the arity of $F)$. Let $X_{\beta}$ and $X_{\gamma}$ be sets of free generators for the $\mathbf{V}$-free algebras $\mathfrak{F}_{\beta}(\mathbf{V})$ and $\mathfrak{F}_{\gamma}(\mathbf{V})$ respectively, and denote by $g$ the surjective $\tau$-homomorphism from $\mathfrak{F}_{\beta}(\mathbf{V})$ to $\mathfrak{F}_{\gamma}(\mathbf{V})$ induced by a surjective function from $X_{\beta}$ to $X_{\gamma}$. Because $\underline{K}$ is an isomorphism and $\mathbf{V}^{\prime}$ has a free algebra $\mathfrak{F}_{\beta}\left(\mathbf{V}^{\prime}\right)$ on $\beta$ generators, we must have $\mathfrak{F}_{\beta}(\mathbf{V})^{\prime}=\mathfrak{F}_{\beta}\left(\mathbf{V}^{\prime}\right)$. Hence $g$ is a surjective $\tau^{\prime}$-homomorphism from $\mathfrak{F}_{\beta}\left(\mathbf{V}^{\prime}\right)$ to $\mathfrak{F}_{\gamma}(\mathbf{V})^{\prime}$ such that $g(x) \in X_{\gamma}$ for all $x \in X_{\beta}$. This clearly implies that $\mathfrak{F}_{\gamma}(\mathbf{V})^{\prime}$ is $\left(\tau^{\prime}-\right)$ generated by $X_{\gamma}$. It follows readily that $\mathfrak{F}_{\gamma}(\mathbf{V})^{\prime}$ is the $\mathbf{V}^{\prime}$-free algebra on $\gamma$ generators.

$(\mathbf{d})^{\prime \prime} \Rightarrow(\mathbf{a})^{\prime \prime} \quad \mathbf{V}^{\prime}$ having a free algebra $\mathfrak{F}_{\gamma}\left(\mathbf{V}^{\prime}\right)$ on a set $X_{\gamma}=\left\{x_{\mu}\right\}_{\mu \in \gamma}$ of $\gamma$ generators, the fact that $\underline{K}$ is an isomorphism (by II) implies that $\underline{K}\left(\mathfrak{F}_{\gamma}(\mathbf{V})\right.$ ) $\left(=\mathfrak{F}_{\gamma}(\mathbf{V})^{\prime}\right)=\mathfrak{F}_{y}\left(\mathbf{V}^{\prime}\right)$. Now, the elements of $\mathfrak{F}_{\gamma}\left(\mathbf{V}^{\prime}\right)$ are the usual equivalence classes of the $\gamma$-ary $\tau^{\prime}$ - terms, and this implies that $\mathbf{V} \vDash \forall \mathbf{x}(F(\mathbf{x})=t(\mathbf{x}))$ for some $\tau^{\prime}$-term $t$ and $\mathbf{x}=\left\langle x_{\mu}\right\rangle_{\mu \in \gamma}$.

$(\mathbf{a})^{\prime \prime} \Rightarrow\left(\mathbf{c}_{1}\right)^{\prime \prime}$ This is proven in much the same way than $(\mathbf{a})^{\prime} \Rightarrow(c)^{\prime}$ in II.

$\left(c_{1}\right)^{\prime \prime} \Rightarrow\left(c_{2}\right)^{\prime \prime}$ From $\left(c_{1}\right)^{\prime \prime}$, we have that $\mathbf{V}^{\prime}=\operatorname{Var}\left(\mathbf{V}^{\prime}\right)$, and hence $\mathbf{V}^{\prime}$ is closed under unions of $\alpha$-sequences. By II, this implies $\left(c_{2}\right)^{\prime \prime}$.

$(\mathbf{h})^{\prime \prime} \Rightarrow(\mathbf{a})^{\prime \prime}$ Let $X_{\gamma}$ be a set of free generators for $\mathfrak{F}_{\gamma}(\mathbf{V}) \quad(\gamma$ the arity of $F)$. It is easily seen that the $\left(\tau^{\prime}-\right)$ subalgebra of $\mathfrak{F}_{\gamma}(\mathbf{V})^{\prime}$ generated by $X_{\gamma}$ is the $\operatorname{Var}\left(\mathbf{V}^{\prime}\right)$-free algebra on $X_{y}$. The fact that it extends to a $(\tau-)$ subalgebra of $\mathfrak{F}_{\gamma}(\mathbf{V})$ implies that $\mathfrak{F}_{\gamma}(\mathbf{V})^{\prime}=\mathfrak{F}_{\gamma}\left(\mathbf{V}^{\prime}\right)$. As in the proof of $(\mathbf{d})^{\prime \prime} \Rightarrow(\mathbf{a})^{\prime \prime}$, this gives the result.

The remaining implications follow easily from what we have already proved.

Remarks. (i) The equivalence of $(a)^{\prime \prime}$ and $\left(c_{1}\right)^{\prime \prime}$ is the natural extension of the De Bouvère Definability theorem to equational logic. It was proved, in the case where $\alpha=\omega$, by Weaver (see [10]; also, $(\mathbf{a})^{\prime \prime} \Leftrightarrow(\mathbf{h})^{\prime \prime}$ is his Lemma 1). Note that the corresponding extension of the Beth Definability theorem (the equivalence of (a)" and (b)) fails, as shown in (ii) below. The equivalence of $(\mathrm{a})^{\prime \prime}$ and (e) $)^{\prime \prime}$ in the case where $\alpha=\omega$ was proved by McKenzie [8]. Both 
proofs utilized first-order model theory. The equivalences in II and the present form of the lemma are due to Hébert.

(ii) Consider respectively the classes Grp of all groups $\left(\tau=\left\{\cdot,()^{-1}, 1\right\}\right)$, Mon of all monoids $\left(\tau^{\prime}=\{\cdot, 1\}\right)$ and Sem of all semigroups $\left(\left(\tau^{\prime}\right)^{\prime}=\{\cdot\}=\tau^{\prime \prime}\right)$. Then $\operatorname{Mon}=\operatorname{Var}\left(\mathbf{G r p}^{\prime}\right)$, Sem $=\operatorname{Var}\left(\mathbf{M o n}^{\prime}\right), \underline{K}: \underline{\mathbf{G r p}} \rightarrow \underline{\mathbf{G r p}^{\prime}}$ satisfies II but not III, and $K:$ Mon $\rightarrow$ Mon' satisfies I but not II. In other words, the "forgetful"

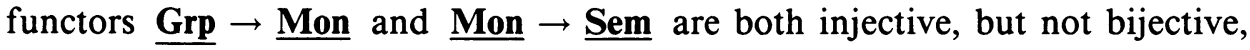
on objects, and only the first one is full. Note however that their composition $\underline{\mathbf{G r p}} \rightarrow \underline{\text { Sem }}$ is full. Hence the functor $\underline{K}: \underline{\mathbf{G r p}} \rightarrow \underline{\mathbf{G r p}}{ }^{\prime \prime}$ does satisfy II: indeed, the sentence $\forall y(y=1 \leftrightarrow y \cdot y=y)$ is true in every group (but not in every monoid).

(iii) If the statements of II are satisfied, then $\mathbf{V}^{\prime}$ has all the "free algebras" in the sense of category theory (i.e. " $U$-free objects", where $U$ is the forgetful functor to the category of sets). But if III is not satisfied, these will not be the "usual" ones, as they are not generated by the subset on which they are free. This occurs for Grp' in (ii) above.

(iv) In II, we do not know if (f) ${ }^{\prime}$ and (g) imply the previous statements for $\alpha \neq \omega$. Closely related is the following open problem: Is any $L_{\alpha}(\tau)$-elementary class of models which is closed under limits necessarily locally $\alpha$-presentable (in the terminology of [5])? That this is true for $\alpha=\omega$ follows from Proposition 16 of [9].

(v) III yields a characterization of equational definability in first-order logic: if $\mathbf{C}$ is an elementary class (i.e. $\mathbf{C}=\mathbf{M}(\mathbf{T H}(\mathbf{C})))$ of $\tau$-algebras and $\tau=\tau^{\prime} \cup\{F\}$, then $\mathbf{C}:=\forall \mathbf{x}(F(\mathbf{x})=t(\mathbf{x}))$ for some $\tau^{\prime}$-term $t$ if and only if the induced mapping $\operatorname{Var}(C) \rightarrow \operatorname{Var}\left(C^{\prime}\right)$ is bijective. In fact, there is no necessity to restrict ourselves to algebraic languages, and this result is equally true if $\tau$ also contains predicate symbols.

(vi) The first author has proven a purely categorical version of the lemma. This leads to results analogous to some of the ones in II and III for situations outside model theory ([6]).

(vii) The situation in $\mathrm{I}(\mathrm{a})$ is usually referred to as " $F$ is explicitely definable in $\mathbf{V}$ (by $\phi(\mathbf{x}, y)$ )". Let $\mathbf{V}_{1}$ and $\mathbf{V}_{2}$ be elementary classes (respectively varieties) of $\tau_{1}$ - and $\tau_{2}$ - algebras respectively, $\tau_{1}$ and $\tau_{2}$ finitary. We say that $\mathbf{V}_{1}$ and $\mathbf{V}_{2}$ are definitionally (respectively polynomially) equivalent provided there is an elementary class (respectively a variety) $\mathbf{C}$ of $\left(\tau_{1} \cup \tau_{2}\right)$-algebras such that all operations in $\tau_{i} \backslash \tau_{j}$ are explicitely definable in $\mathbf{C}$ by $L_{\omega}\left(\tau_{j}\right)$-formulas (respectively are equivalent in $\mathbf{C}$ to $\tau_{j}$-terms), $\{i, j\}=\{1,2\}$. In [4], De Bouvère points out that his [3] shows (using a different terminology) that the definitional equivalence of $\mathbf{V}_{1}$ and $\mathbf{v}_{2}$ amount to the existence of bijective "reduct" mappings $K_{i}: \mathbf{C} \rightarrow \mathbf{V}_{\mathbf{i}}, i=1,2$, from an elementary class $\mathbf{C}$ of $\left(\tau_{1} \cup \tau_{2}\right)$-algebras. Replacing "definitional" by "polynomial" and "elementary class" by "variety," III provides an equational analogue of that result. Whether or not all definitionally equivalent varieties are polynomially equivalent appears to be an open question. However, as a consequence of the remark following the lemma, we 
can show the following: Two varieties $\mathbf{V}_{1}$ and $\mathbf{V}_{2}$ are polynomially equivalent if and only if they are definitionally equivalent through a $\mathbf{C}$ (as above) which is closed under (finite) products (if and only if the associated $K_{1}$ and $K_{2}$ are isomorphisms). Notice also that this will be the case if $\mathbf{V}_{1}$ and $\mathbf{V}_{2}$ are definitional extensions of a same elementary class (i.e. if there exist $\tau \subseteq\left(\tau_{1} \cap \tau_{2}\right)$ and bijective reduct mappings $K_{i}^{\prime}: \mathbf{V}_{\mathbf{i}} \rightarrow \mathbf{C}, i=1,2$, in a $\tau$-elementary class $\left.\mathbf{C}\right)$.

\section{REFERENCES}

1. S. Burris and H. P. Sankappanavar, A course in universal algebra, Springer-Verlag, New York, 1980.

2. C. C. Chang and H. J. Keisler, Model theory, North-Holland, Amsterdam, 1977.

3. K. L. De Bouvère, A mathematical characterization of explicit definability, Indag. Math. 25 (1963), 264-274.

4. __ Synonymous theories, in Symposium on the Theory of Models, North-Holland, Amsterdam, 1965.

5. P. Gabriel and F. Ulmer, Lokal prasentierbare Kategorien, Lecture Notes in Math., no. 221, Springer-Verlag, Berlin, 1971.

6. M. Hébert, On the fullness of certain functors, (to appear in J. Pure Appl. Algebra).

7. J. R. Isbell, Functorial implicit operations, Israel J. Math. 15 (1973), 185-188.

8. R. N. McKenzie, Letter to S. Givant, 1983.

9. H. Volger, Preservation theorems for limits of structures and global sections of sheaves of structures, Math. Z. 166 (1979), 27-53.

10. G. E. Wever, Equational definability, manuscript, March 1987.

Department of Mathematics, McGill University, Montréal, Canada H3A 2K6 and Département de MathématiQues, Université laval, Québec, Canada G1K 7P4

Department of Mathematics, University of California at Berkeley, Berkeley, CALIFORNIA 94720

Department of Philosophy, Bryn Mawr College, Bryn Mawr, Pennsylvania 19010

Current address (Michel Hébert): Ecole Polytechnique, Université des Sciences et Techniques de Masuku, B. P. 941, Franceville, Gabon 